\title{
Why Cytoskeletal Associated Proteins are Important in Colorectal Cancer Patients: Molecular and Bioinformatic Analysis
}

\section{Kolorektal Kanser Hastalarında Hücre İskeletiyle Illişkili Proteinler Neden Önemlidir: Moleküler ve Biyoinformatik Analiz}

\section{Dilara Fatma Akın Balı', (ㄷ) Didem Torun Özkan²}

'Department of Medical Biology, Niğde Ömer Halisdemir University Faculty of Medicine, Niğde, Turkey

${ }^{2}$ Medical Laboratory Programme, İstanbul Okan University Vocational School of Health Service, İstanbul, Turkey

\begin{abstract}
Introduction: It has been aimed to analyze the role of pathogenic effects of mutation and expression anomalies occurring on diaphanous-related formin 1 (DIAPH1), WASP actin nucleation-promoting factor (WASP), myosin heavy chain 9 (MYH9), actinin alpha 1 (ACNT1), filamin A (FLNA), and tubulin beta 1 class VI (TUBB1), which are known as fundamental cellular skeleton proteins, on the development and progression of cancer via bioinformatic tools.

Methods: The genome sequence and expression profiles of 594 Colorectal Cancer (CRC) patients were obtained via bioinformatic tools, which provide data for The Cancer Genome Atlas. The mutation patterns of six genes were determined in detail, and for the prediction of pathogenic properties of identified changes for CRC, Polymorphism Phenotyping v2, Screening for Non-Acceptable Polymorphisms, and the Catalogue Of Somatic Mutations In Cancer were utilized. Apart from the mutation profile, the effects of existing mutations on messenger ribonucleic acid (mRNA) expression and survival were also identified. Moreover, the Search Tool for the Retrieval of Interacting Genes/Proteins network analysis was realized to further comprehend the functional relations of proteins in cellular processes.

Results: There have been 142 distinct point mutations, gene amplification, and deep deletions identified on DIAPH1, WAS, MYH9, ACNT1, FLNA, and TUBB1 genes. ACTN1 and FLNA low mRNA expression levels for DIAPH1 increased, and the mRNA expression level was statistically significant $(p<0.05)$. Prognosis-wise, the effect of mRNA expression on survival in the absence of disease was meaningful for FLNA $(p=0.011)$.

Discussion and Conclusion: Bioinformatic analysis data in DIAPH1, WASP, MYH9, ACNT1, FLNA, and TUBB1 genes, which are important in CRC pathogenesis revealed in this study, will be a guide for future laboratory studies.

Keywords: Cytoskeleton; Gene Expression; Mutation; Colorectal Cancer
\end{abstract}

\begin{abstract}
Cite this article as: Akin Bali DF, Torun Ozkan D. Why Cytoskeletal Associated Proteins are Important in Colorectal Cancer Patients: Molecular and Bioinformatic Analysis. Lokman Hekim Health Sci 2021;1(1):14-14-31.
\end{abstract}

Correspondence: Dilara Fatma Akin-Bali, PhD. Department of Medical Biology, Nigde Omer Halisdemir University, Faculty of Medicine, Nigde, Turkey E-mail: dilarafatmaakin@gmail.com Submitted:08.07.2021 Accepted: 27.07.2021 
$T^{h}$ he impairment or loss of several aspects of normal cellular activity is the common entity of all cancer types. However, the genomic instability engendered using the defects that cause tissue degeneration on cellular morphogenesis, migration, the achieving of the ability of invasion, and anomalies in mitosis also accompany the progression of the disease. ${ }^{[1]}$ The cytoskeleton proteins that are composed of different subcategories of proteins including microtubules, actin, and intermediate filaments are vital for the survival and cellular processes of normal and cancer cells. ${ }^{[2-4]}$ At the same time, the cytoskeleton network at the cellular transformation phase can be reprogrammed to assist the progression of cancer via stimulating survival, growth, and invasion of the tumor cell. Therefore, the tumor cells gain various properties. ${ }^{[3-6]} \mathrm{CRC}$ is a heterogeneous disease that causes the colon and rectum mucosa to transform into an invasive cancer that develops because of genetic, epigenetic, and peripheral factors. ${ }^{[7,8]}$ The interactions between the adhesion complexes and cytoskeleton proteins are crucial for the epithelial structure of a normal intestine to survive. The anomalies in the expression and functional activity of the cytoskeleton proteins accompanied by the deterioration of the epithelial homeostasis are known to be the precursors in cancer commencement and progression. ${ }^{[6,7,9]}$ We aim to contribute to the development of potential objectives toward new molecular biomarkers and anticancer medicine by realizing the comprehensive molecular analysis of fundamental cytoskeleton proteins of ACTN1, MYH9, TUBB1, FLNA, DIAPH1, and WASP, whose effects on CRC pathogenesis are not completely discovered, by revealing the potential anomalies on a group of CRC patient group. In this study, we planned the investigation of the effects of the relations, which is influential on CRC malignant cell transformation between the mutations and representation irregularities in their expressions on genomic instability, invasion, and increment of metastasis abilities.

\section{Materials and Methods}

\section{Study Group}

TCGA CRC (n:526) dataset was downloaded via cBioPortal, and the demographic, clinic, and genetic information was exhibited in Table 1. The data used in our study were obtained from the public database TCGA; therefore, ethical approval was not required.

\section{Analysis of Mutation Profile}

Cbio Cancer Genomics Portal is a bioinformatic tool that contains and provides the mutation data, copy number alterations, microarray and RNA sequencing-based mRNA expression alterations, deoxyribonucleic acid (DNA) methylation data, and protein and phosphoprotein levels from the TCGA database. ${ }^{[10]}$ Oncoprint, Mutasyon, and Coexpression tabs were utilized to examine the mutations of DIAPH1, WASP, MYH9, ACNT1, FLNA, and TUBB1 genes on the $C R C$ patients. The type and frequency of the mutations were identified for each gene individually.

\section{Prediction of Pathogenic Mutations}

The potential effects of the missense mutations that were identified on DIAPH1, WASP, MYH9, ACNT1, FLNA, and TUBB1 genes were analyzed through two pathogenicity estimators. Polymorphism Phenotyping v2: It is a versatile bioinformatic tool that estimates the potential structural and functional consequences of Mutation/Single Nucleotide polymorphisms on amino acid position. It provides the estimation results between the score interval of 0.0 (benign) and 1.0 (potentially damaging). ${ }^{[1]}$ The Catalogue Of Somatic Mutations In Cancer: It is a comprehensive source for the research of the characteristics of somatic mutations and their effects. The pathogenic effects of the identified mutations are determined through the database using the Functional Analysis through Hidden Markov Models algorithm. This algorithm estimates the functional, molecular, and phenotypical effects of the missense mutations. ${ }^{[12]}$

\section{Gene Expression Profile and Survival Analyses}

GEPIA (Gene Expression Profiling Interactive Analysis) is an interactive bioinformatic tool that was developed to realize the differential expression analysis on tumor or normal tissues, generate profiles with respect to the cancer types or pathological phases, patient survival analysis, analog gene detection, correlation analysis, and other customizable analyses. ${ }^{[13]}$ The effects of mRNA expression levels of DIAPH1, WASP, MYH9, ACNT1, FLNA, and TUBB1 genes and high and low mRNA expression levels on overall and disease-free survival were realized comparatively on the healthy control group and the CRC ( $\mathrm{n}: 275)$ patient group. The analysis of the genes that were predicted by cBioPortal was realized.

\section{Protein-Protein Interaction Analysis}

The Search Tool for the Retrieval of Interacting Genes/Proteins (STRING) database was applied to evaluate the protein-protein interaction information. The estimated interactions between DIAPH1, WASP, MYH9, ACNT1, FLNA, and TUBB1 proteins and the protein net that defines the direct (physical) and indirect (functional) relations among the proteins were generated and analyzed using the STRING database. ${ }^{[14]}$ 


\section{Statistical Analyses}

All the statistical analyses that were used to evaluate the study data were realized using the GEPIA bioinformatic tool. ${ }^{[13]}$ The survival analysis that we accomplished on the GEPIA database was realized via the exploitation of KaplanMeier curves. The log-rank test was applied to investigate the effects of low and high expression groups on survival. The Spearman test was used by the database for correlation studies. In all the studies, $\mathrm{p}<0.05$ was determined as the statistically significant value.

\section{Results}

\section{Results of Mutation Profile Analysis}

There have been 142 different genetic alternations that were identified on DIAPH1, WASP, MYH9, ACNT1, FLNA, and TUBB1 genes among 526 CRC patients. Of these alterations, 117 were missense mutation, three were nonsense mutation, eight were frameshift mutation, 11 were splice region mutation, and one was gene fusion mutation. Moreover, although deep deletion due to homozygote loss was identified on WASP and ACTN1 genes, gene amplification anomalies were detected on WAS, MYH9, FLNA, and TUBB1 genes.

All the genetic alterations given in Appendix 1 are shown with detailed explanations. The probability of hosting a genetic alteration were DIAPH1, 2.9\%; WAS, 1.9\%; MYH9, 8\%; ACTN1, 3\%; FLNA, 7\%; and TUBB1, 7\%. The highest number of alterations occurred on MYH9 (51 mutations), whereas the least number of alterations were detected on TUBB1 (five mutations). It is statistically meaningful that MYH9;FLNA, DIAPH1;MYH9, and DIAPH1;ACTN1 hosted genetic alterations together $(p=0.016, p=0.025$, and $p=0.042)$. The localization of mutations detected on the domains of proteins belonging to the study genes is shown in Figure 1 as a representation.

The frameshift mutations that were originated from the shifting of the reading frame that has the capability of causing early termination of polypeptides were detected on DIAPH1, WAS, MYH9, and FLNA genes. The nonsense mutation that gives birth to stop codons was identified on MYH9 and FLNA genes. Because of the splice mutations' presence on the bases of splice region, which is completely preserved during the evolutionary process, the unfunctional transcripts containing the intron information originated and anomalies in the expression occurred. Consequently, a loss of functionality is probable. DIAPH1, ACTN1, MYH9, and FLNA gene mutations were detected on metastatic patients and presented in Appendix 1.
Table 1. Summary of demographic, clinical, and genetic data in CRC patients

Characteristics

Patient data n:594 (\%)

Gender

Male/Female/NA

$312 / 282 / 2$

Diagnosis age, years

Race category

White

$285(48)$

Black or African American

$64(10.8)$

Asian

$12(2.0)$

NA

American Indian

232 (39.1)

Tumor type

Colon adenocarcinoma

$1(0.2)$

Rectal adenocarcinoma

378 (63.6)

Mucinous adenocarcinoma

$155(26.1)$

61 (10.3)

Sample type

Primary

594

Overall survival status

Living

471

Deceased

120

NA

3

Tumor stage code

$\begin{array}{lc}\text { T1 } & 20(3.4) \\ \text { T2 } & 103(17.3) \\ \text { T3 } & 401(67.5) \\ \text { T4 } & 30(5.1) \\ \text { T4a } & 27(4.5) \\ \text { T4b } & 10(1.7) \\ \text { NA } & 3(0.5) \\ \text { etastatic stage code } & \\ \text { M0 } & 440(74.1) \\ \text { M1 } & 69(11.6) \\ \text { MX } & 62(10.4) \\ \text { M1A } & 11(1.9) \\ \text { M1B } & 3(0.5) \\ \text { NA } & 9(1.5)\end{array}$

\begin{tabular}{lc}
\hline Alteration frequency & Case (Frequency \%) \\
\hline DIAPH1 mutation & $15(2.9)$ \\
WAS mutation & $7(1.3)$ \\
MYH9 mutation & $38(7.2)$ \\
ACTN1 mutation & $15(2.9)$ \\
FLNA mutation & $36(6.8)$ \\
TUBB1 mutation & $4(0.76)$ \\
WAS amplification & $1(0.19)$ \\
MYH9 amplification & $1(0.19)$ \\
FLNA amplification & $2(0.38)$ \\
TUBB1 amplification & $34(6.46)$ \\
WAS deep deletion & $2(0.38)$ \\
ACTN1 deep deletion & $1(0.19)$ \\
\hline
\end{tabular}

NA: Not Applicable; MX: Distant Metastasis Cannot Be Assessed; M0: No Distant Metastasis; M1: Distant Metastasis; T: Tumor. 


\section{MYH9 Analysis}

MYH9 codes a protein addressed as muscle exterior myosin IIA (NMMIIA) of $453 \mathrm{kDa}$ mass in the hexametric form responsible for cell locomotion, megakaryocyte contraction, and the preservation of cytoplasmic matrix. MYH9 gene has four major domains that are called head domain (GHD), neck, coiled-coil tail domain (TD), and non-helical tail domain. ${ }^{[15]}$ In our study, we have identified 20 different mutations that code nonmuscle myosin type IIA (NMMIIA) heavy chain in the region between exons 2 and 19 on the GHD. We identified 39 distinct mutations in the TD region, which are coded by exons $21-40$. There was one missense mutation identified in the Neck region, which is coded by exon 20 .

\section{TUBB1 Analysis}

There are two important domains, namely, Guanosine Triphosphate (GTP) domain and Microtubule-Associated Protein (MAP), that are present in TUBB1 protein, which comprised 451 amino acids. ${ }^{[16]}$ Out of seven mutations that were detected, especially p.V169L and p.R77Q mutations reside on the GTP domain.

\section{FLNA Analysis}

FLNA gene integrins interact with transmembrane receptor complexes and secondary messengers to code prevalently expressed filamin A'l of 280 kDA mass actin-binding protein responsible for the rearrangement of the cytoskeleton and realize signal transmission, cell migration, and adhesion. It has four major domains, namely, An N-terminal F-Actin-Binding Area, which comprised two tandem calponin homology $(\mathrm{CH})$ regions $(\mathrm{CH} 1$ and $\mathrm{CH} 2$ ), two ROD regions composed of repetitions similar to $23 \mathrm{lg}$, and a repetition region on the C-terminal that passes through dimerization before the interaction with the membrane receptors. ${ }^{[17]}$ We have identified 44 distinct mutations on the FLNA gene, and these mutations reside on the domains coded by exons $2-48$.

\section{WASP Analysis}

WASP gene is responsible for the coding of Wiskott-Aldrich syndrome protein that takes part in the signal transmission from the cell surface receptors to the actin cytoskeleton composed of 502 amino acids. It is composed of N-terminal Ena-VASP homology domain 1 (EVH1), GTPaz bonding domain, poly-proline domain (PPPP), and verproline homology (V) and C-terminal domain composed of central (C) cluster. ${ }^{[18]}$ Out of the seven mutations that were detected,
p.A139V and p.R138Q mutations reside on the EVH1 domain; p.X311 splice region mutation resides on the PPPP domain starting point; and p.P353Hfs*92 mutation, which has the potential to change the reading frame, reside on the PPPP domain.

\section{DIAPH1 Analysis}

DIAPH1 belongs to the formin family and has two functional regions: formin homology 1 and 2 regions ( $\mathrm{FH} 1$ and $\mathrm{FH} 2$, respectively) and guanosine triphosphatase bonding area and dimerization region and several regulatory domains. ${ }^{[19]}$ Out of 18 mutations we identified, three distinct frameshift mutations were present on $\mathrm{FHI}$ and $\mathrm{FH} 2$ domains.

\section{ACTN1 Analysis}

There is an effective binding domain of two $\mathrm{CH}$ regions, four spectrin (SPEC) motives, and two calcium bonding EF hands (EFh), and a calponin-like motive (CaM) is present on the N-terminal of ACTN1 protein. On the C-terminal, two EFh (efhand Ca_insen) are indifferent to degenerate calcium domains residue. ${ }^{[20]}$ The missense mutations of p.F99V and p. R133H15, which are responsible for actin bonding functionality on the $\mathrm{CH}$ domain, were of 15 identified mutations. There was also a p.X254_splice mutation identified in the region between the $\mathrm{CH}$ domain and SPEC motives.

\section{In Silico Pathogenicity-Estimated Results of Mutations}

According to our estimated pathogenetic test, out of the 142 identified mutations on target genes, 117 of them were in the missense mutation character. With respect to the output scores from the two analysis programs, 52 missense mutations were pathogenic. The pathogenicity scores are provided in detail in Appendix 1.

\section{Results of Gene Expression and Survival Analyses}

In the light of the analyses results of the high and low mRNA expression levels of DIAPH1, WASP, MYH9, ACNT1, FLNA, and TUBB1 genes of 275 CRC patients, the mRNA expression level of the DIAPH1 gene was higher than the healthy group expression levels of ACTN1 and FLNA, which were detected to be low, which is statistically meaningful (Fig. 2). When a comparison was made on WAS, MYH9, and TUBB1 genes for the healthy group, there was not any discrepancy identified. With respect to the results of our survival analysis, it has been shown that mRNA expression levels have no significant effect on the survival rate. However, when the mRNA expression levels 


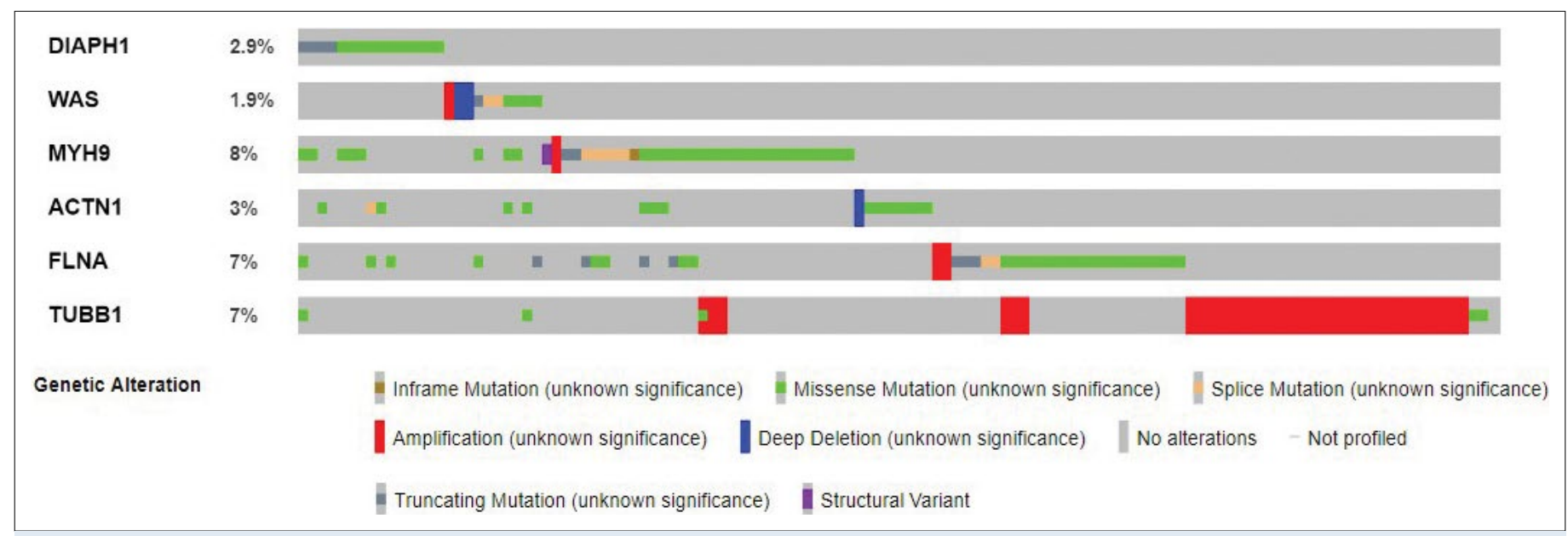

Figure 1. Lollipop diagrams of DIAPH1, WAS, MYH9, ACNT1, FLNA, and TUBB1 mutations detected in 594 patients with CRC patients.
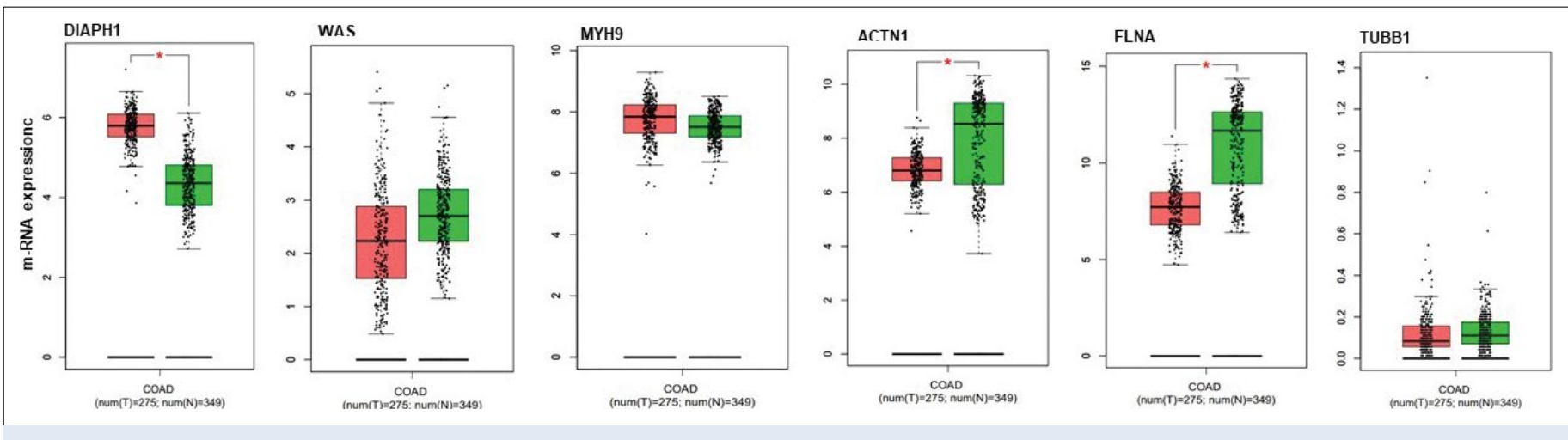

Figure 2. Box plot representation of the comparative mRNA expression level of DIAPH1, WAS, MYH9, ACNT1, FLNA, and TUBB1 genes among CRC patients and healthy tissue samples using GEPIA; the red box indicates cancer tissue, and the green box indicates healthy tissue.

*: Indicates that the results are statistically significant.

were evaluated on the survival rate of disease-free conditions, it has been validated that high mRNA level is advantageous when compared with low mRNA level in terms of the length of the survival without disease, which is statistically significant ( $p=0.011$ ) (Fig. 3). All the six genes exhibited positive correlation in our correlation analysis with the scope of understanding the comparative analysis of the effects of the association of DIAPH1, WASP, MYH9, ACNT1, FLNA, and TUBB1 mutations and APC mutation on mRNA expression level. ACTN1-APC, DIAPH1-APC, FLNA-APC, WASP-APC, and MYH9-APC coexpression analysis showed a positive correlation $(p=0.01, p=3.78 \mathrm{e}-16$, $\mathrm{p}=1.828 \mathrm{e}-3, \mathrm{p}=7.984 \mathrm{e}-4$, and $\mathrm{p}=1.49 \mathrm{e}-9$ ) (Fig. 4).

\section{Results of the Protein-Protein Interaction Analysis}

STRING analysis was applied to establish the estimated protein interactions net of the DIAPH1, WASP, MYH9, ACNT1, FLNA, and TUBB1 genes. With respect to the analysis, 10 proteins were reported of which all the proteins in relation. It has been seen that mutations exist for every gene that codes the 10 proteins, which is in interaction with DIAPH1, WASP, MYH9, ACNT1, FLNA, and TUBB1 genes that were depicted in Figure 5 in our CRC patient group. In the perspective of proteins that have mutual interaction with our target proteins, CDC5L protein with DIAPH1, TUBB1, and MYH9 proteins have interaction. $C D C 5$ protein is a cell cycle regulator located at the Gap2 (G2)/Mitosis (M) passage, and it is known that it involves in the process of mending DNA damages and mRNA splicing. The CRC patient group has a mutation (2.4\%) in their CDC5L gene.

\section{Discussion}

Colorectal cancer has the third-highest incidence rate, and it is the culprit behind one-fourth of all cancer deaths worldwide. Although its etiology and pathogenesis are not completely understood, the environmental, ethnic, economical, and genetic factors play a major role in the progression of 

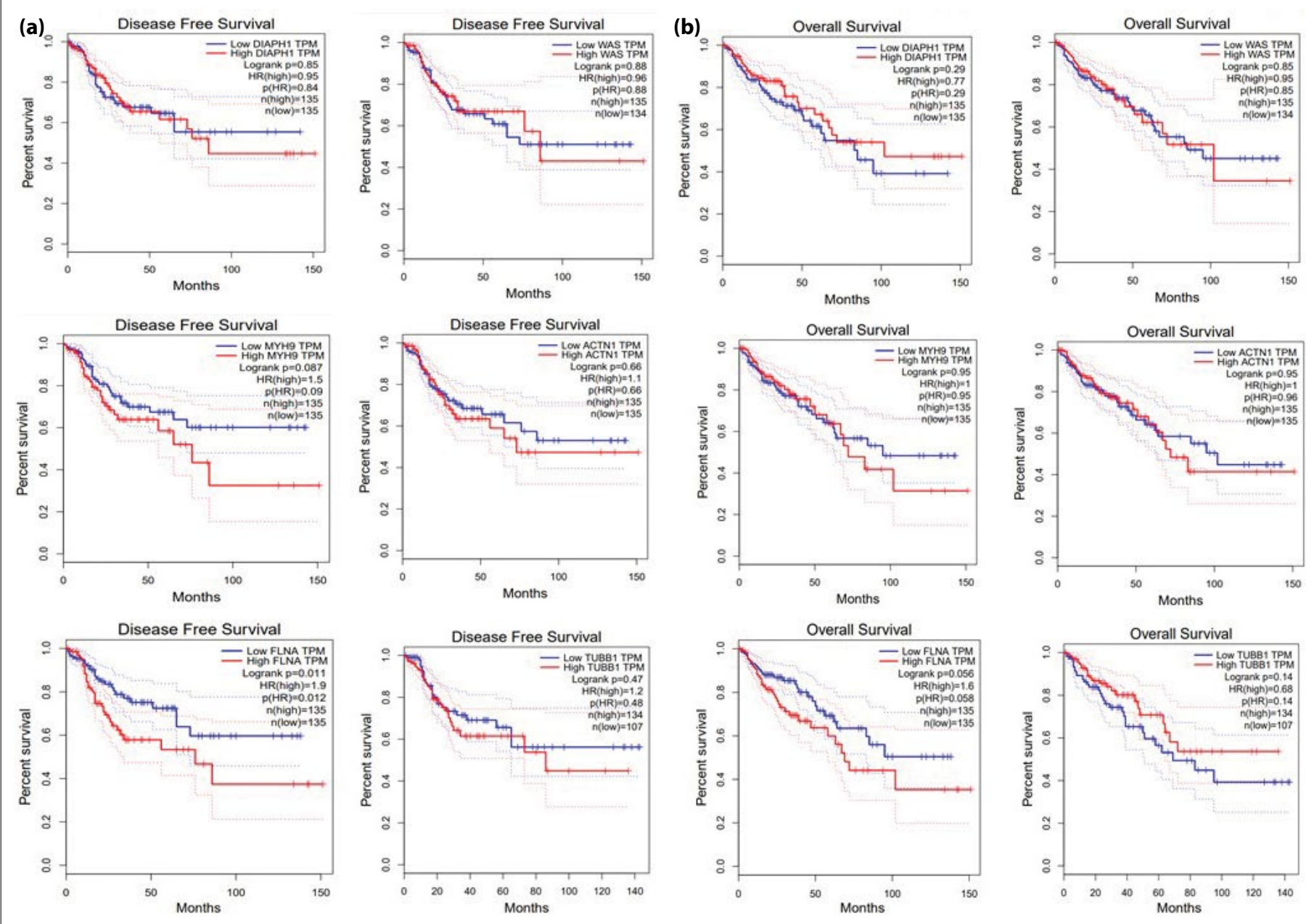

Figure 3. Comparison of the Kaplan-Meier survival curves of the high and low expressions of DIAPH1, WAS, MYH9, ACNT1, FLNA, and TUBB1 in CRC patients $(p<0.05)$.

CRC. ${ }^{[7,8,21]}$ We have realized comparative and joint comprehensive analyses of mutation and mRNA expression profiles of cytoskeleton proteins DIAPH1, WASP, MYH9, ACNT1, FLNA, and TUBB1, which are known to be effective in the pathogenesis of CRC but not completely enlightened, through various online tools. First, the genome sequences of 594 CRC patients were gathered from the cbiO platform, which provides TGCA datasets. Then, the mutation profiles regarding DIAPH1, WASP, MYH9, ACNT1, FLNA, and TUBB1 were analyzed. In our study, we have identified a total of 142 mutations (117 missense mutations, three nonsense mutations, eight frame shift mutations, 11 splice region mutations, one gene fusion, deep deletion, and gene amplification) regarding the six genes in 594 CRC patients. The highest number of mutations were detected on MYH9, whereas the least number of mutations were present on WASP.

MYH9 regulates cancer progression, and it is known to be functional as oncogenic and tumor-suppressing, but its part in CRC is not completely understood yet. ${ }^{[22]}$ Globular head domain is responsible for actin bonding and force generation via MgATPaz activation. ${ }^{[15]}$ On this domain, out of 20 identified mutations 2 (p.G205=,p.X290_splice) of them resided on the splice region. Since these mutations change the p.X290_splice region, which we determined as pathogenic, it will likely result as a passive transcript. It enhances the motion generated by the conformational alterations of the neck domain motor and acts as a lever that operates as a bonding region for tenuous chains using two IQ motives. ${ }^{[15]}$ Because of its pathogenic character, p.K833N mutation exacerbates the function of its domain. Owing to its oncogenic properties, it has been unveiled that it supports tumor formation by regulating MAPK/AKT signalization of MYH9. ${ }^{[15,22]}$ The p.Y9H mutation we detected on the globular domain has shown to be in gain characteristics. Moreover, it is probable that accompanied by the MYH9 gene amplification, it can gain more functionalities. However, we have revealed that mRNA expression level is no different than 


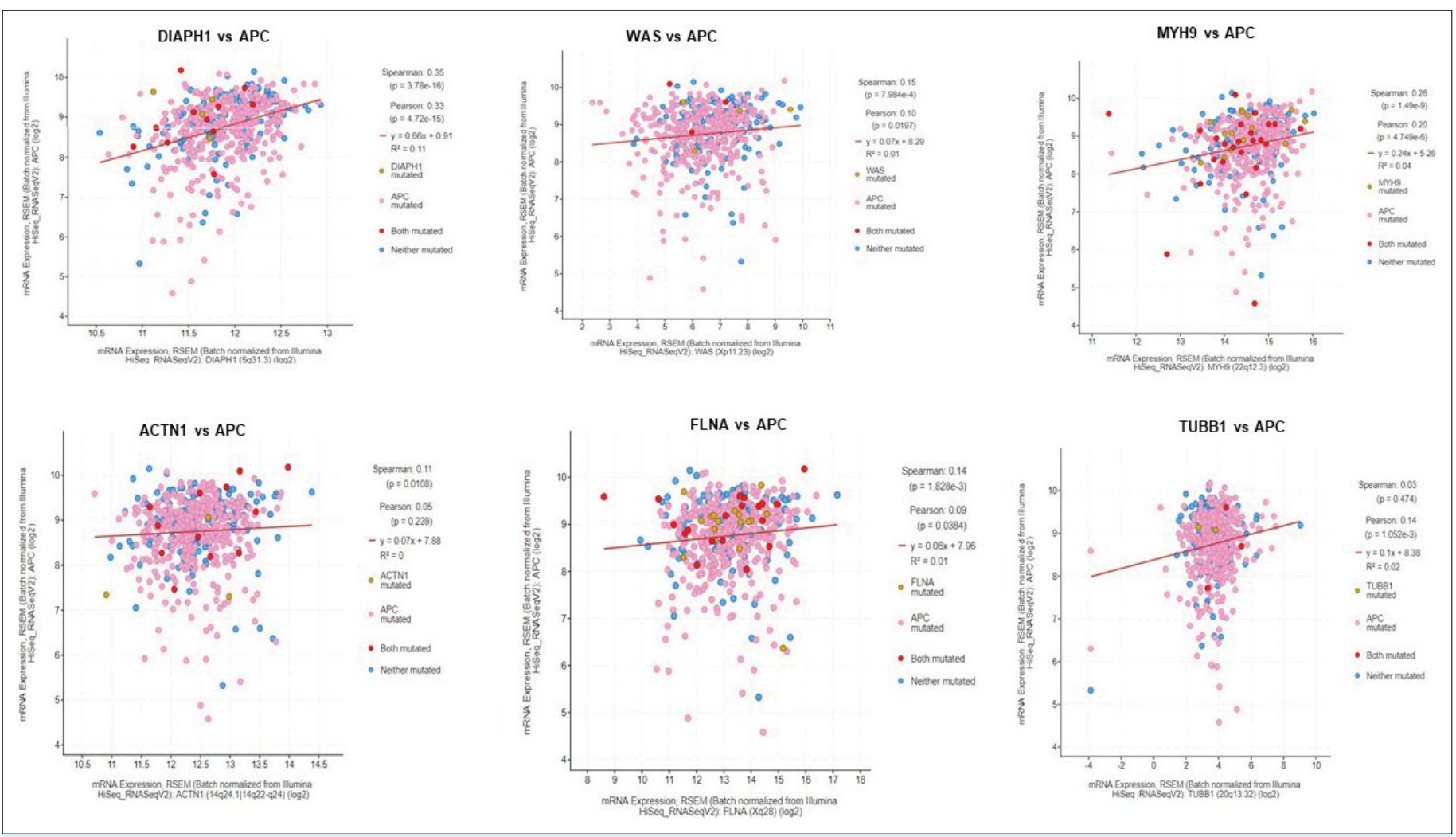

Figure 4. APC mutation and the effect of target gene mutations on normalized gene expression.

that of healthy tissues and it has no power on survival. Microtubules are ubiquitous cytoskeleton units that take place in maintaining the cell morphology, intracell transportation, cell signalization, cell locomotion, and various cellular functionalities including mitosis. ${ }^{[16]}$ It can cause genetic inconsistency and DNA damage accumulation of TUBB1 dysfunction. In our study, this statement has been shown to be conserved. It can cause the chaotic activation of the GTP domain in which p.V169L mutation has rich mRNA expression. Moreover, an association of cell cycle regulator $\mathrm{CDC} 5 \mathrm{~L}$ p.K487N and with TUBB1 p.R77Q mutations are pathogenic. It has been proven that ACTN1 is related to the negative prognosis in breast cancer, carcinoma with oral squamous, and acute lymphoblastic leukemia; however, there has been no research on the investigation of its role in $\mathrm{CRC}^{[23]}$ In our findings, the ACTN1 mRNA expression levels in cancer tissues are low; still, no significant effects of this case have been found in common and disease-free survival. Nonetheless, because of the presence of p.X254 splice mutation on the splice region, it has been shown that a pathogenic character hinders the production of the functional transcript of 892 amino acid length. The presence of short nonfunctional transcripts might probably be the reason behind the significant scarcity in mRNA expression. It has been shown that $A B$ domain mutations induce increased ACTN1 bonding in the actin filaments. The four SRs domain constitutes the rod area of ACTN1 protein. ${ }^{[20,23]}$ On these SPEC-like repeats 1-4 (SR1-SR4) domains, we have identified nine distinct missense mutations. SR1-SR4 domains are the central region in which actin dimers originate. Four of the missense mutations we detected were identified as pathogenic using the estimation program. Hence, we hypothesize that by impeding the generation of actin dimers, they might cause protein imbalance in the seconder helical structure.

FLNA is interrelated to many cellular functions such as cell signalization, motility, phosphorylation, proteolysis, ion channel regulation, transcription regulation, receptor activation, and muscle development. ${ }^{[17]}$ It has been reported in a few studies that the chemotherapy sensitivity of FLNA can be exploited as a cancer predictor and prognosis biomarker. ${ }^{[17,24]}$ However, the clinical representation of FLNA in CRC and its biological function is controversial. FLNA's abnormal representation in many types of cancers such as breasts cancer, colon cancer, melanoma, and prostate cancer or its mutation has been reported. ${ }^{[17,24]}$ In the previous studies, FLNA's having low expression in CRC and relation to the pathogenesis have been indicated. Parallel to the literature, the mRNA expression level of FLNA in cancer tissues was found to be statistically insignificant in our study, and high FLNA expression has been shown to be effective 


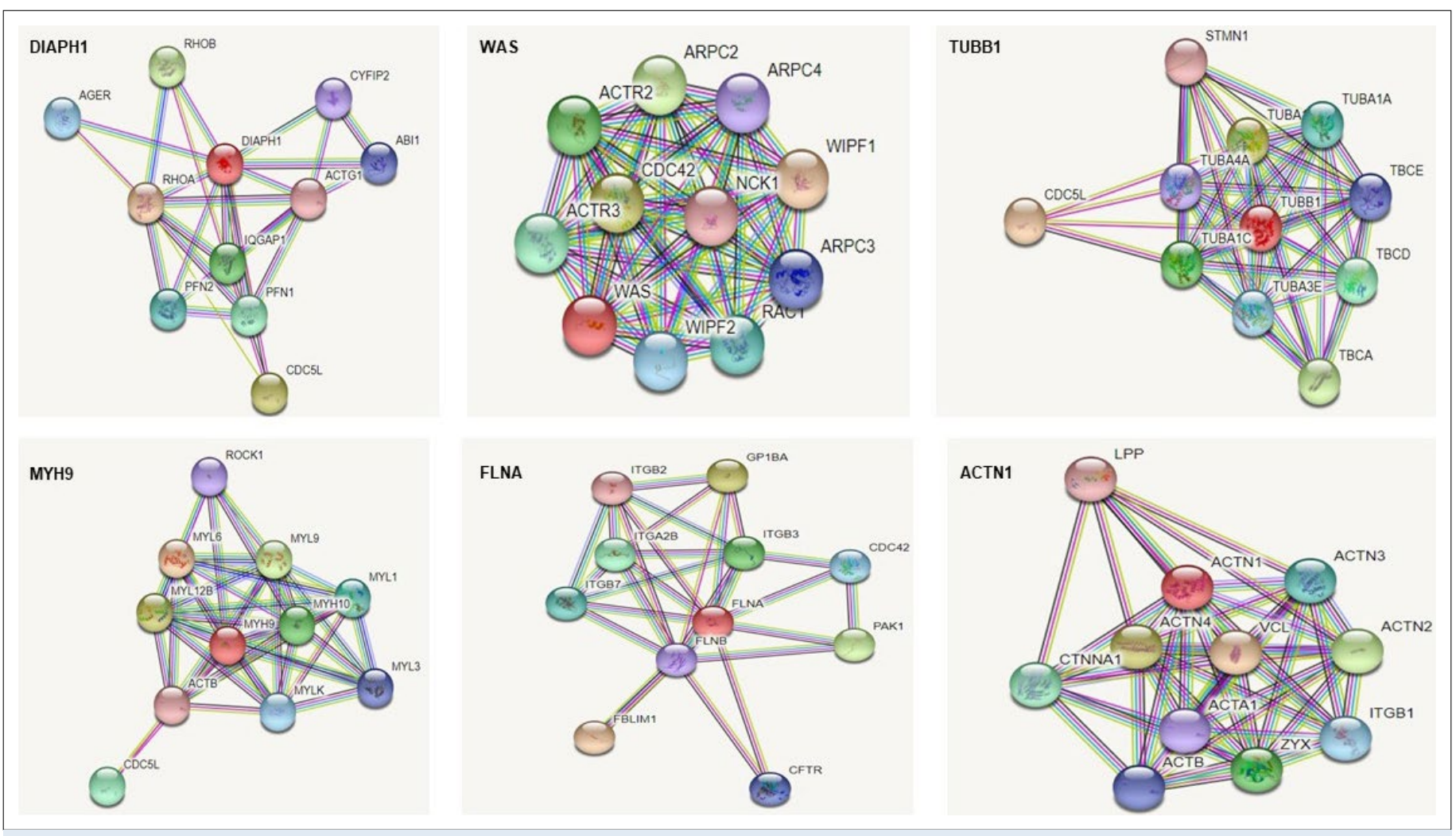

Figure 5. DIAPH1, WAS, MYH9, ACNT1, FLNA, and TUBB1 protein-protein interaction networks. The figure was created via the cBioPortal website.

on disease-free survival. p.X1070_splice, p.X713_splice, and p.X1533_splice mutations and p.R2419* nonsense mutation have the potential to cause short, nonfunctional transcripts responsible for the low expression level.

WASP is a key actin regulatory protein accountable for the control of actin polymerization, adhesion, contraction, and preservation of the cell morphology. It generates the membrane protrusions that are seen during the metastasis and invasion of cancer cells. ${ }^{[18]}$ In our study, WASP mutations in patients with metastasis were not determined. However, the p.A139V and p.R138Q mutations we identified are in the WH1 domain. These mutations are known to have pathogenic characteristics. Mutations in this domain have been reported to destabilize the protein's electrostatic force and protein-protein interactions. ${ }^{[18]}$ The presence of p.X259_ splice mutation, which is responsible for the creation of filopodia and CDC-42 affinity on a base with a preserved splice region, might prevent the generation of a transcript. Located on the proline-rich domain, p.P353Hfs*92 frameshift domain responsible for WASP protein and Arp 2/3 complex interaction is in the pathogenic character and can deteriorate the function of the domain.

DIAPH1 is the actin nucleator and is also bonded to the microtubules with high affinity. The frameshift mutations that reside on $\mathrm{FH} 1-\mathrm{FH} 2$ domain with active actin bonding property might reduce the actin bonding capability by generating a stop codon in the early phase of the polypeptide of 1263 amino acid residues. ${ }^{[19,25]}$ Although the high levels of DIAPH 1 mRNA are a boosting factor for metastasis progression, the advent of resistance against chemotherapy medicine might lead to a negative prognosis.

Epithelial cells in CRC lose their actin cytoskeleton formation and normal cell-to-cell adhesion properties once they become invasive. Further research must allow the dissolving of new cytoskeleton functionalities and the development of novel therapeutic strategies based on the manipulation of the pertinent molecules. Dynamic actin cytoskeleton characterizes normal epithelial cells, polymerization and depolymerization of actin filaments, and alterations of the cells in the process of migration and mitosis. The interactions between adhesion complexes and cytoskeleton proteins are vital for maintaining the structure of epithelial cell structure and allows for the individual cells to respond to different stimuli and signals in harmony. In our study, as a result of a comprehensive molecular approach, we have identified the target gene mutations and expression abnormalities, which we believe to have a negative impact on the aforementioned epithelial net system. Our results constitute a 
preliminary dataset that can be exploited in all the further in vivo and in vitro clinic research aimed at cancer diagnosis and development of new anticancer medicine. Certain restrictive factors are impeding our research in which we investigated the effects of cytoskeleton proteins in CRC pathogenesis. This is because the study was conducted by using bioinformatic tools with a limited experimental setting. Therefore, wet laboratory work is mandatory to reveal the roles of DIAPH1, WASP, MYH9, ACNT1, FLNA, and TUBB1 genes behind $C R C$ pathogenesis.

Acknowledgements: The data used in our study are obtained from public database the TCGA Research Network:

https:// http://www.cancer.gov/tcga www.cancer.gov/tcga. We thank the TCGA, GEPIA, cbio Portal and STRING databases for the availability of the data.

Ethical approval and ethical standards: The data used in our study were obtained from public database TCGA, therefore, ethical approval was not required. Availability of data and materials: The data sets generated and analyzed during the current study are available in TGCA database (https://www.cancer.gov/tcga),The cBio cancer genomics portal(http://www.cbioportal.org/).

Peer-review: Externally peer-reviewed.

Authorship Contributions: Concept: DFAB, DTO; Design: DFAB; Supervision: DFAB, DTO; Data Collection or Processing: DFAB; Analysis or Interpretation: DFAB; Literature Search: DFAB, DTO; Writing: DFAB, DTO; Critical Review: DFAB, DTO

Conflict of Interest: None declared.

Financial Disclosure: The authors declared that this study received no financial support.

\section{References}

1. Ferguson LR, Chen H, Collins AR, Connell M, Damia G, Dasgupta $\mathrm{S}$, et al. Genomic instability in human cancer: Molecular insights and opportunities for therapeutic attack and prevention through diet and nutrition. Semin Cancer Biol 2015;35 Suppl(Suppl):S5-S24.

2. Hall A. The cytoskeleton and cancer. Cancer Metastasis Rev 2009 (1-2);28:5-14.

3. Yilmaz M, Christofori G. EMT, the cytoskeleton, and cancer cell invasion. Cancer Metastasis Rev 2009;28(1-2):15-33.

4. Lens SMA, Medema RH. Cytokinesis defects and cancer. Nat Rev Cancer 2019;19:32-45.

5. Aseervatham J. Cytoskeletal Remodeling in Cancer. Biology (Basel) 2020;9(11):385.

6. Jones MC, Zha J, Humphries MJ. Connections between the cell cycle, cell adhesion and the cytoskeleton. Philos Trans R Soc Lond B Biol Sci 2019;374(1779):20180227.

7. Buda A, Pignatelli M. Cytoskeletal network in colon cancer: from genes to clinical application. Int J Biochem Cell Biol 2004;36(5):759-65.
8. Dekker E, Tanis PJ, Vleugels JLA, Kasi PM, Wallace MB. Colorectal cancer. Lancet 2019;394(10207):1467-80.

9. Ong MS, Deng S, Halim CE, Cai W, Tan TZ, Huang RY, et al. Cytoskeletal Proteins in Cancer and Intracellular Stress: A Therapeutic Perspective. Cancers (Basel) 2020;12(1):238.

10. Cerami E, Gao J, Dogrusoz U, Gross BE, Sumer SO, Aksoy BA, et al. The cBio cancer genomics portal: an open platform for exploring multidimensional cancer genomics data. Cancer Discov 2012;2(5):401-4.

11. Adzhubei I, Jordan DM, Sunyaev SR. Predicting functional effect of human missense mutations using PolyPhen-2. Curr Protoc Hum Genet 2013;Chapter 7:Unit7.20.

12. Tate JG, Bamford S, Jubb HC, Sondka Z, Beare DM, Bindal N, et al. COSMIC: the catalogue of somatic mutations in cancer. Nucleic Acids Res 2019;47(D1):D941-7.

13. Tang Z, Li C, Kang B, Gao G, Li C, Zhang Z. GEPIA: a web server for cancer and normal gene expression profiling and interactive analyses. Nucleic Acids Res 2017;45(W1):W98-102.

14. Szklarczyk D, Gable AL, Lyon D, Junge A, Wyder S, Huerta-Cepas J, et al. STRING v11: protein-protein association networks with increased coverage, supporting functional discovery in genome-wide experimental datasets. Nucleic Acids Res 2019;47(D1):D607-13.

15. Pecci A, Ma X, Savoia A, Adelstein RS. MYH9: Structure, functions and role of non-muscle myosin IIA in human disease. Gene 2018;664:152-67.

16. Schwer HD, Lecine P, Tiwari S, Italiano JE Jr, Hartwig JH, Shivdasani RA. A lineage-restricted and divergent beta-tubulin isoform is essential for the biogenesis, structure and function of blood platelets. Curr Biol 2001;11(8):579-86.

17. Kolahi KS, Mofrad MR. Molecular mechanics of filamin's rod domain. Biophys J 2008;94(3):1075-83.

18. Lane J, Martin T, Weeks HP, Jiang WG. Structure and role of WASP and WAVE in Rho GTPase signalling in cancer. Cancer Genomics Proteomics 2014;11(3):155-65.

19. Lin YN, Windhorst S. Diaphanous-related formin 1 as a target for tumor therapy. Biochem Soc Trans 2016;44(5):1289-93.

20. Pollard TD, Borisy GG. Cellular motility driven by assembly and disassembly of actin filaments. Cell 2003;112(4):453-65.

21. Liu J, Zhang Y, Li Q, Wang Y. Transgelins: Cytoskeletal associated proteins implicated in the metastasis of colorectal cancer. Front Cell Dev Biol 2020;8:573859.

22. Wang B, Qi X, Liu J, Zhou R, Lin C, Shangguan J, et al. MYH9 Promotes Growth and Metastasis via Activation of MAPK/AKT Signaling in Colorectal Cancer. J Cancer 2019;10(4):874-84.

23. Fukumoto M, Kurisu S, Yamada T, Takenawa T. a-Actinin-4 enhances colorectal cancer cell invasion by suppressing focal adhesion maturation. PLoS One 2015;10(4):e0120616.

24. Tian ZQ, Shi JW, Wang XR, Li Z, Wang GY. New cancer suppressor gene for colorectal adenocarcinoma: filamin A. World J Gastroenterol 2015;21(7):2199-205.

25. Yang J, Zhou L, Zhang Y, Zheng J, Zhou J, Wei Z, et al. DIAPH1 is upregulated and inhibits cell apoptosis through ATR/p53/ Caspase-3 signaling pathway in laryngeal squamous cell carcinoma. Dis Markers 2019;2019:6716472. 


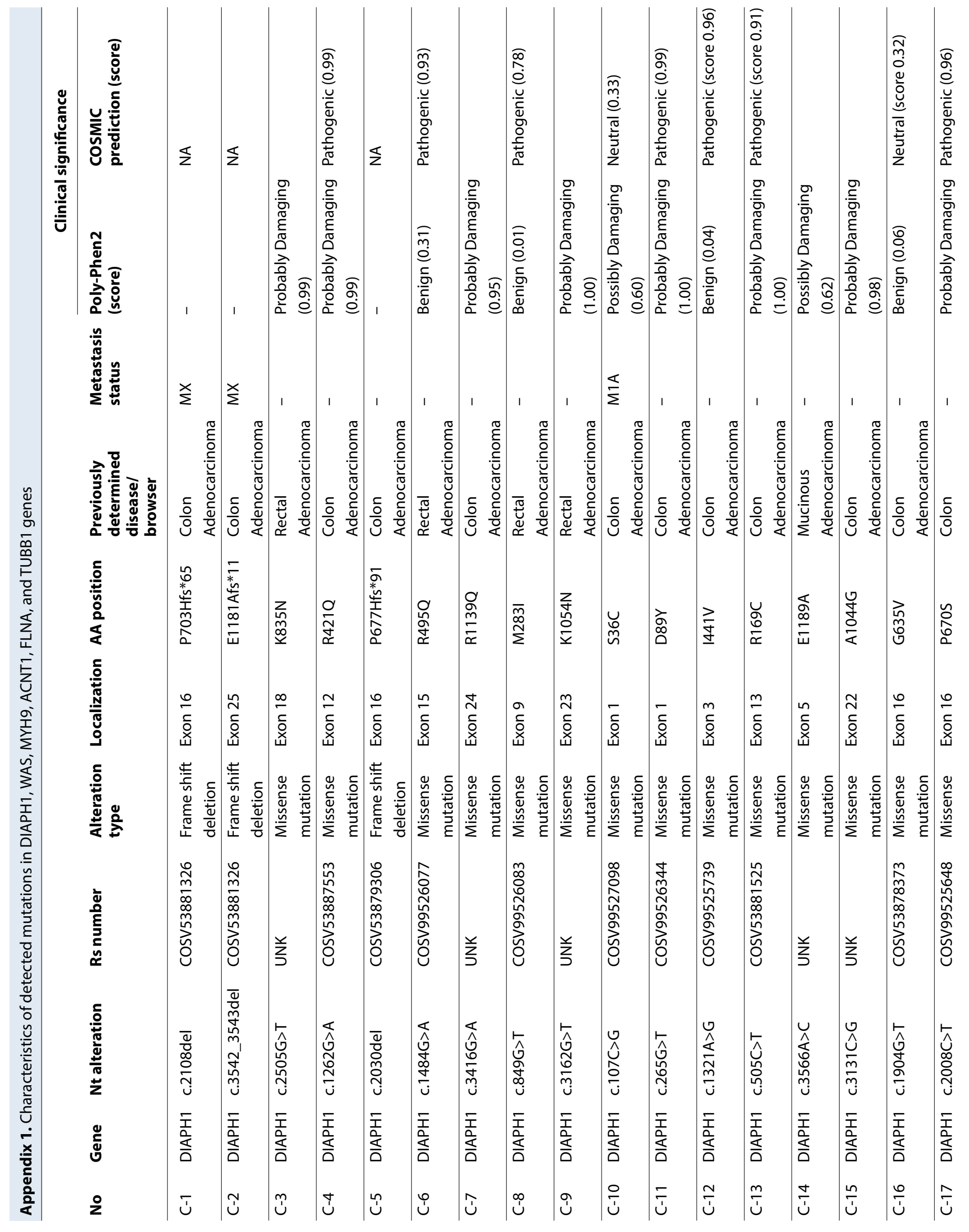




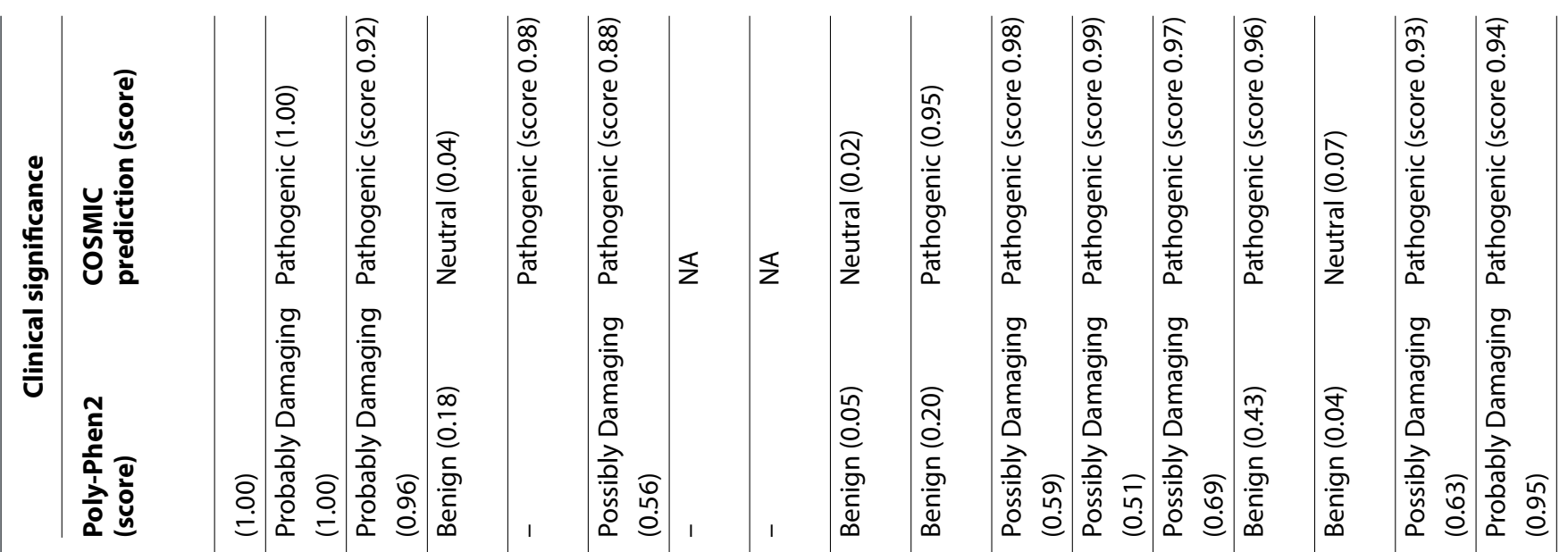

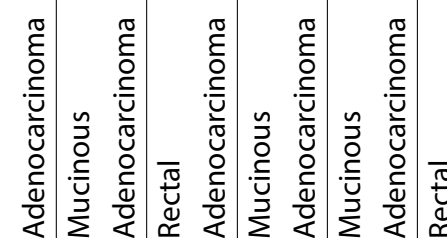

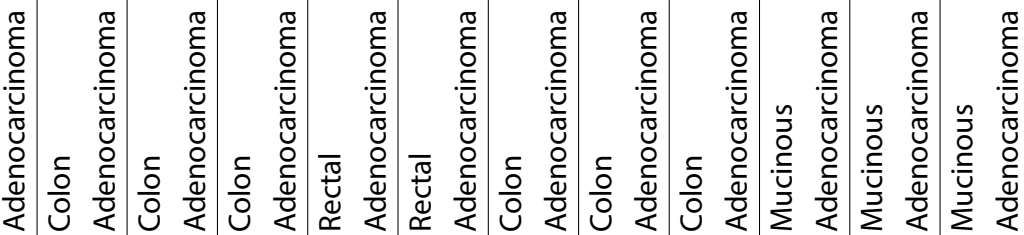

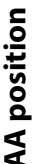

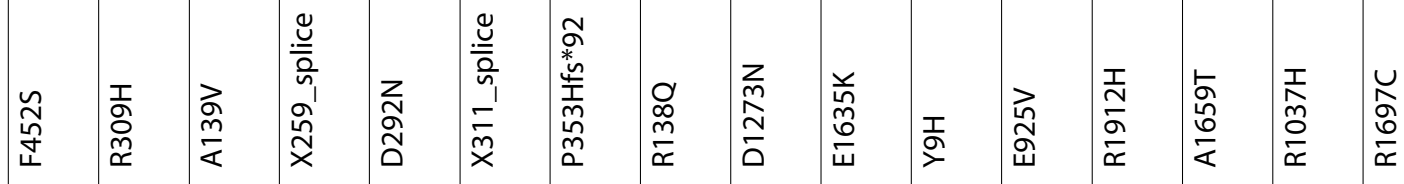

苞

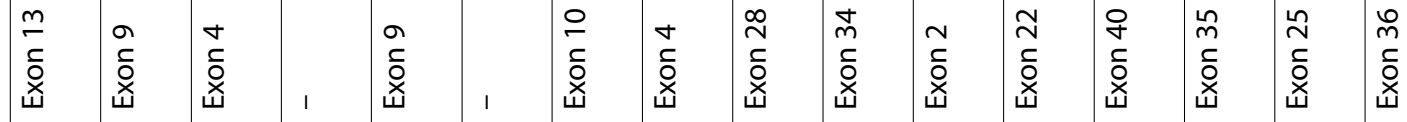

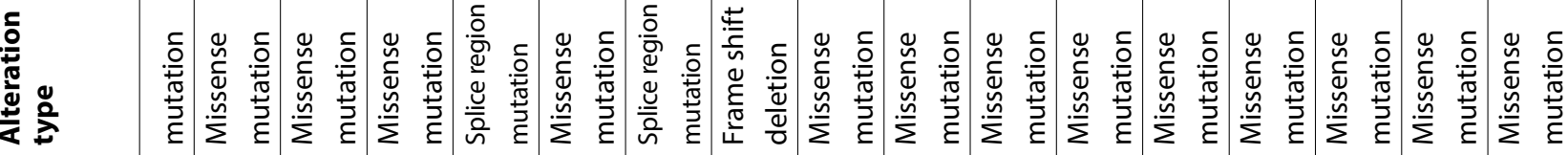

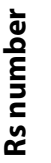

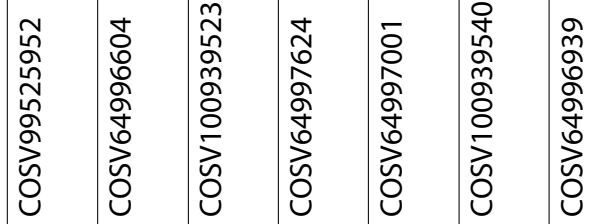

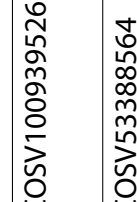

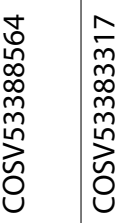

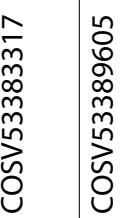

黛

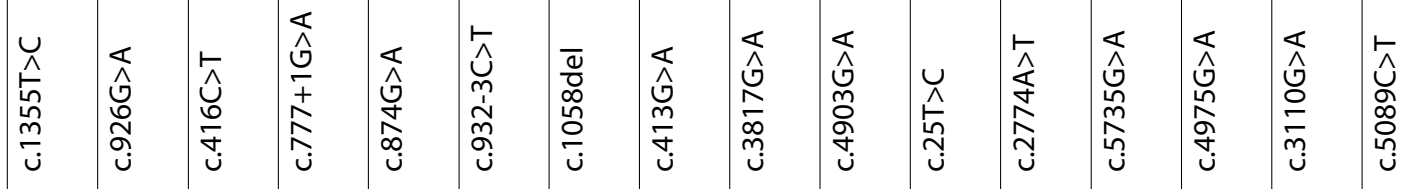

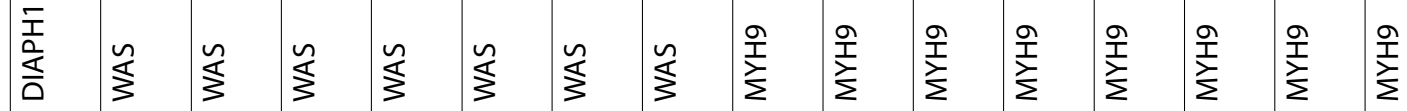

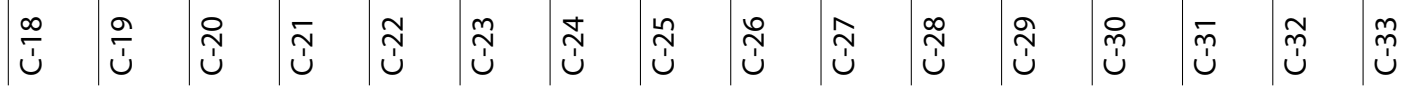




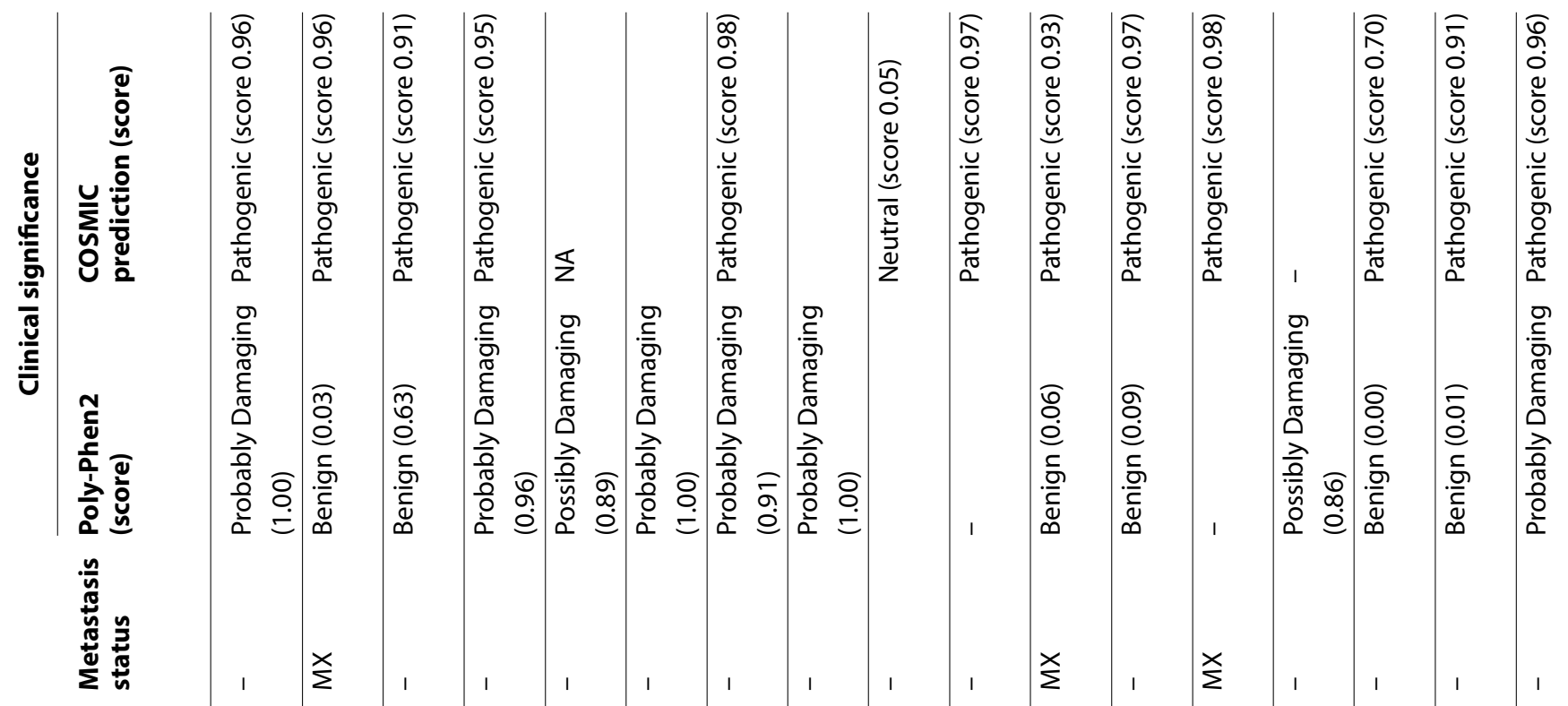

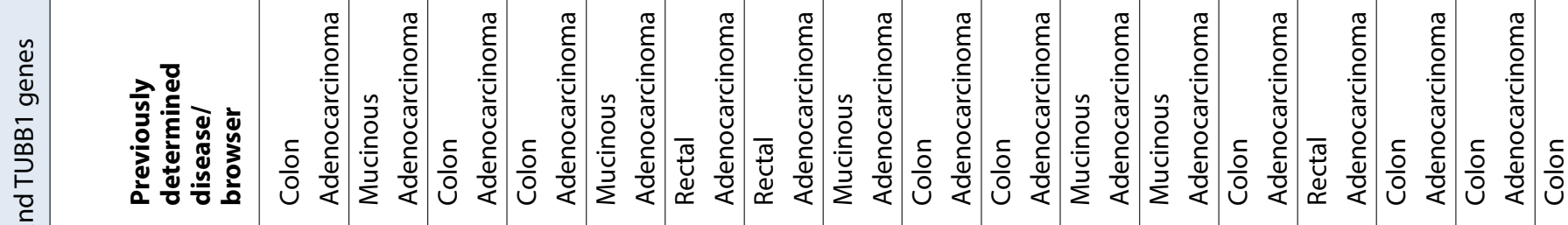

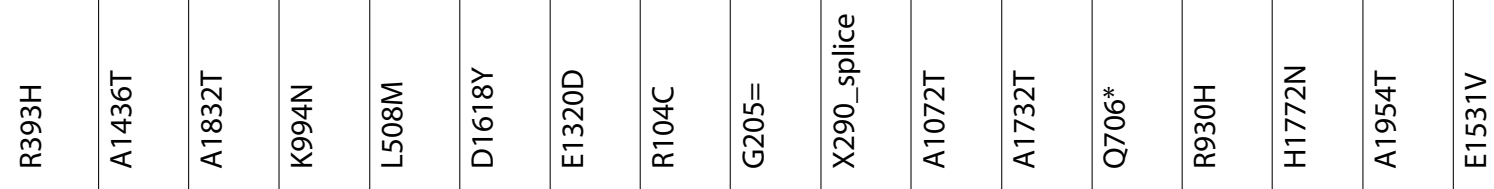

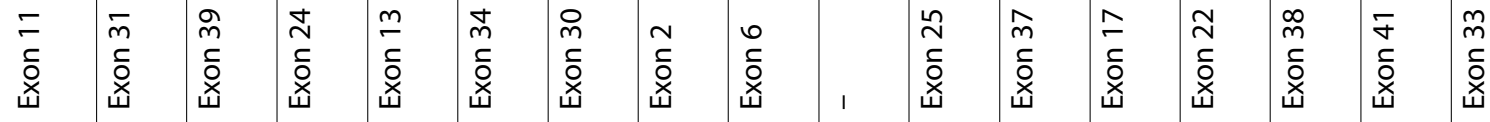

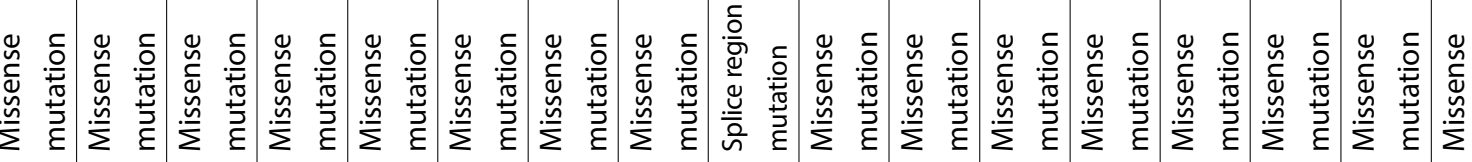

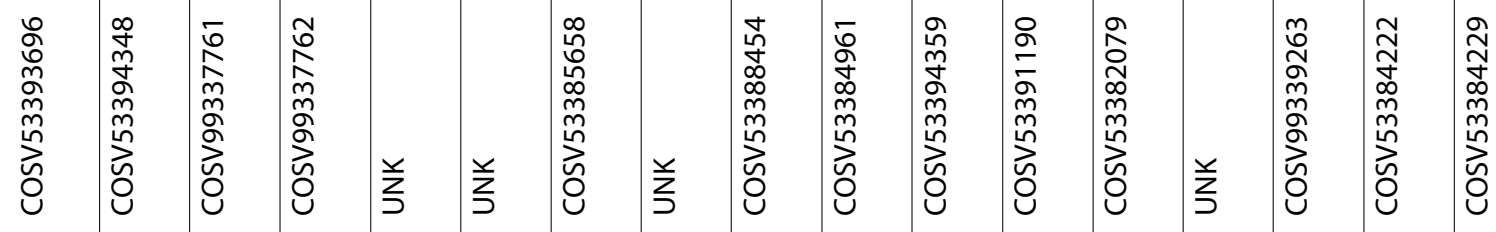

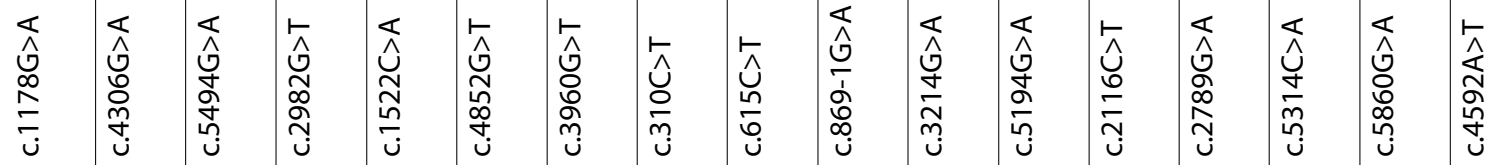

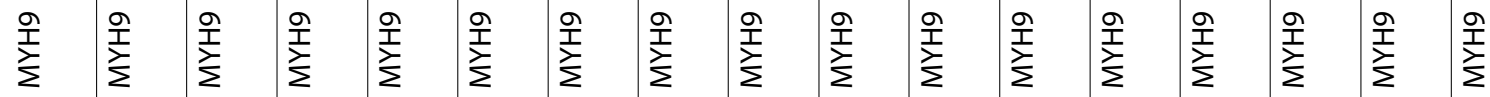

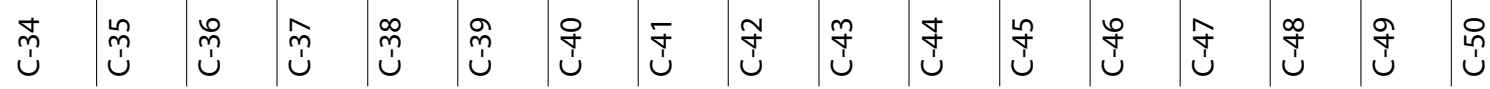




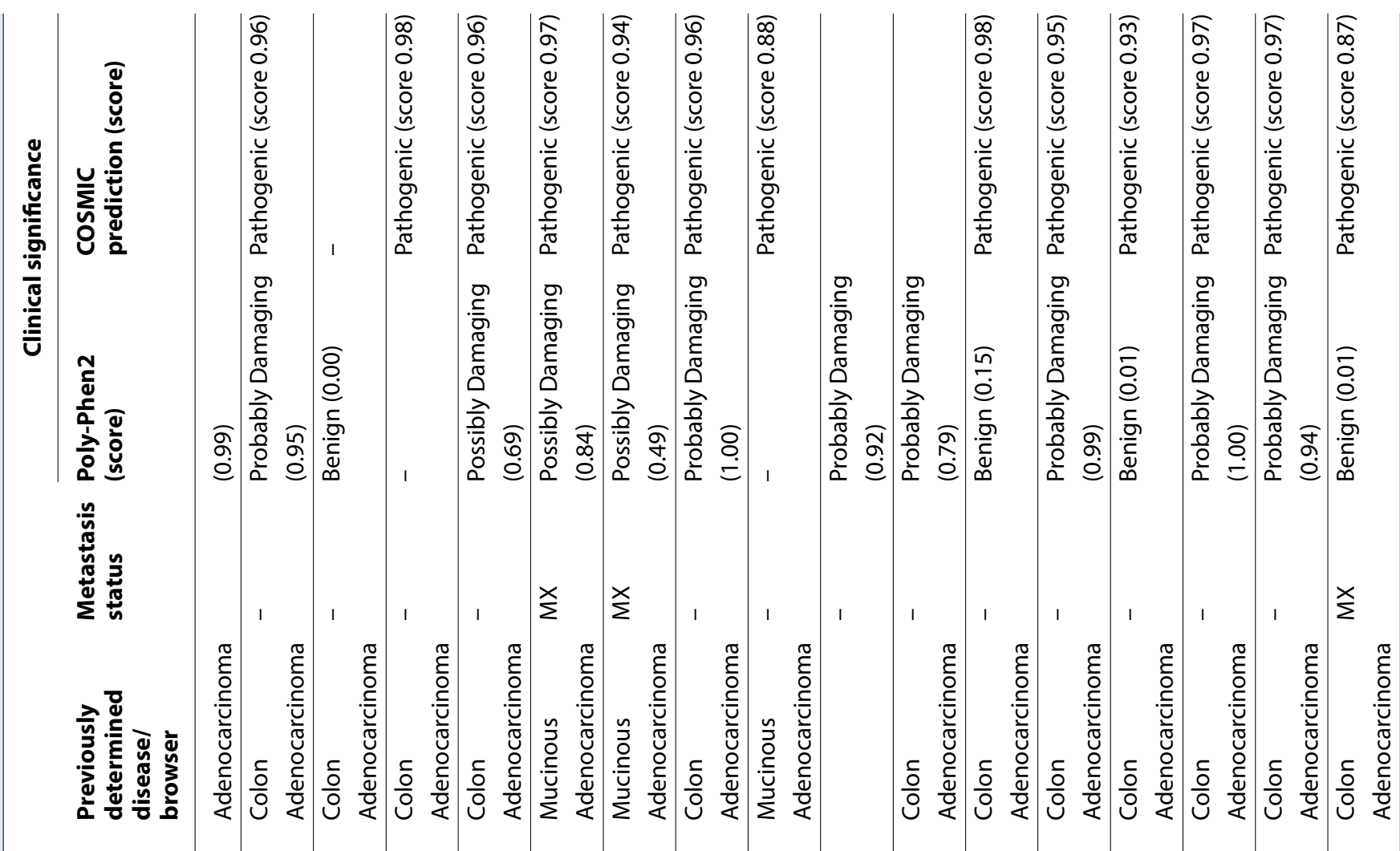

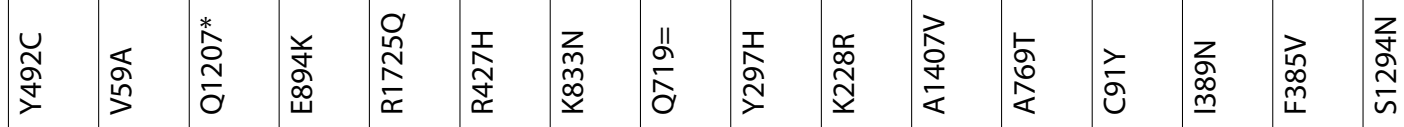

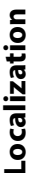

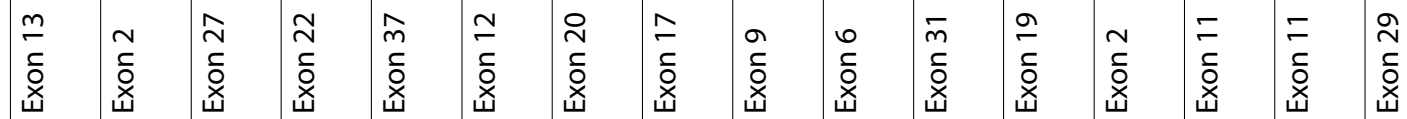

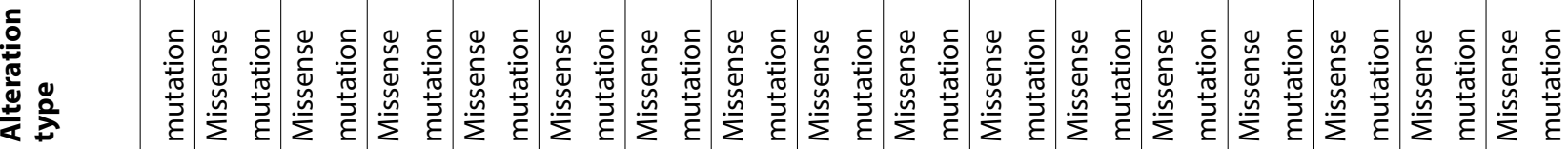

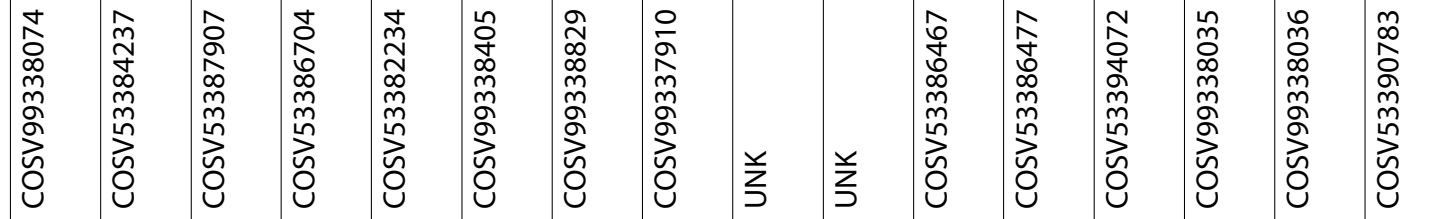

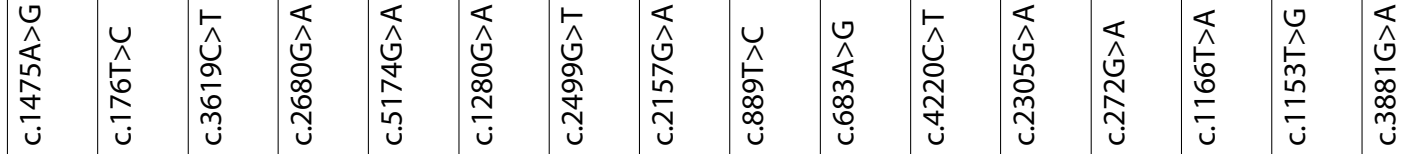

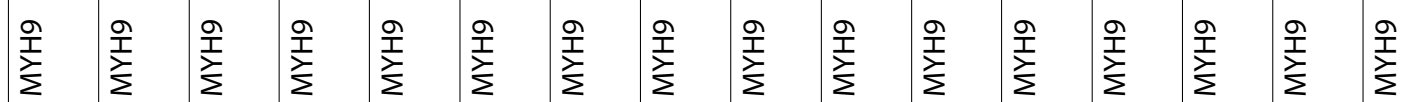

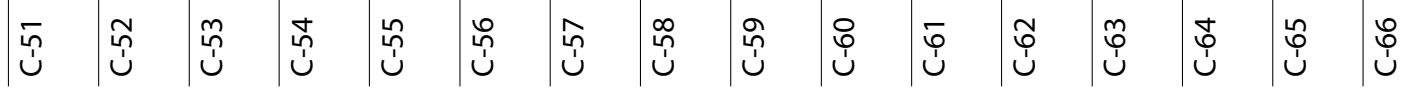




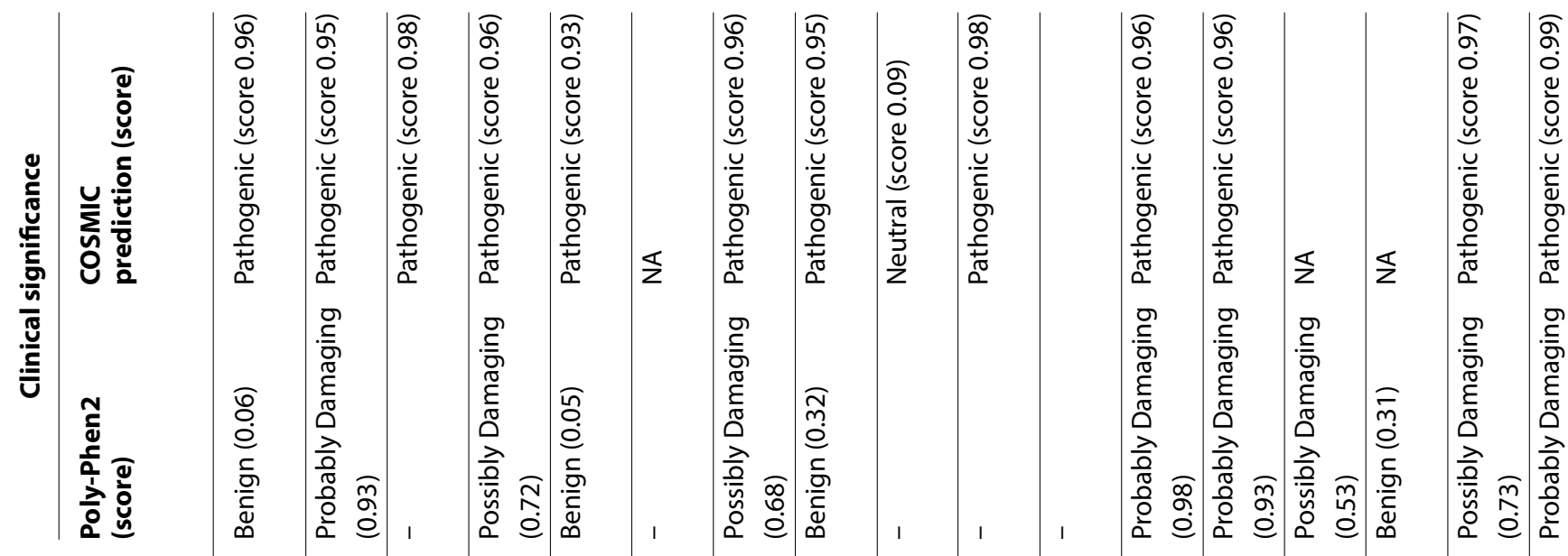

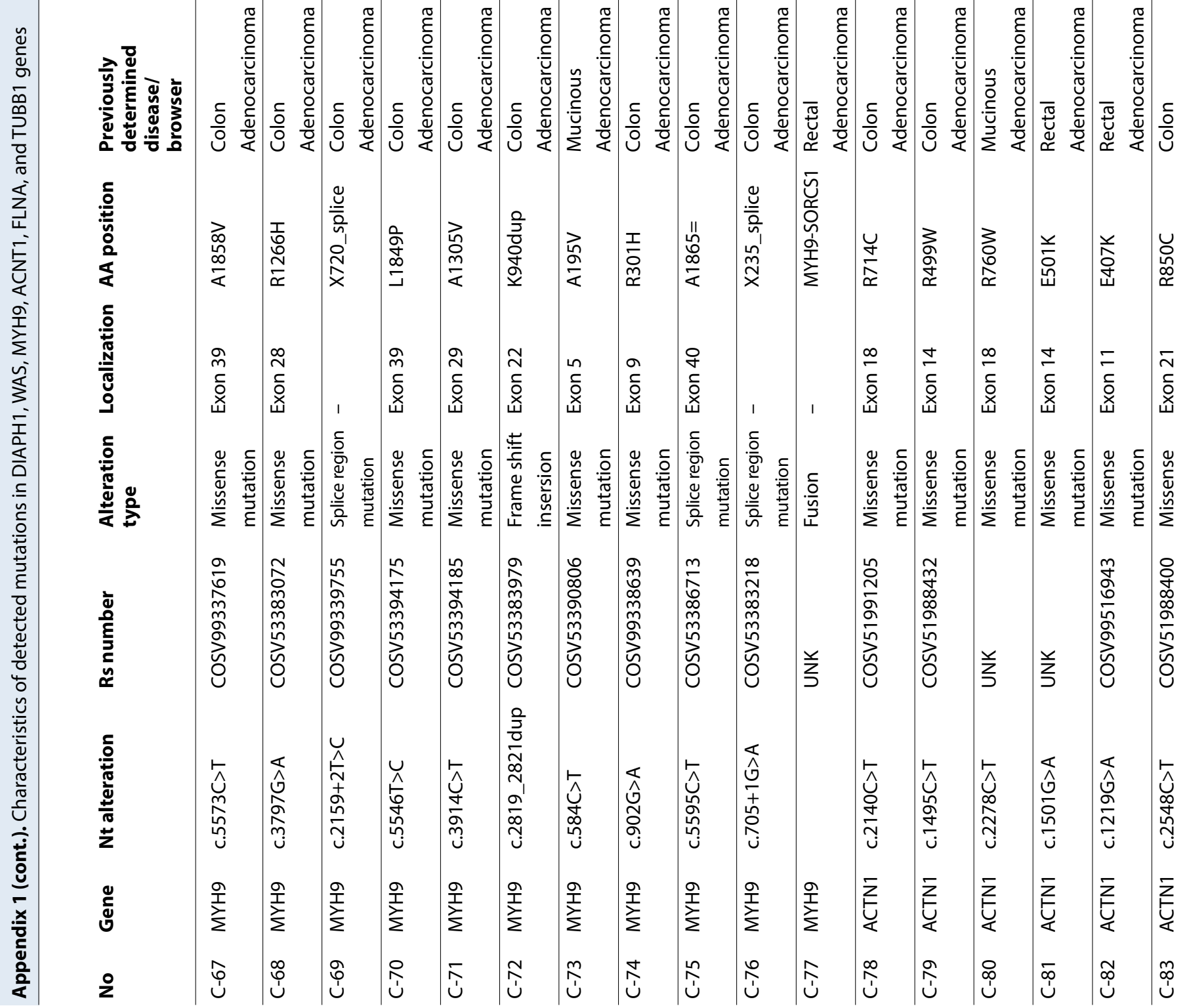




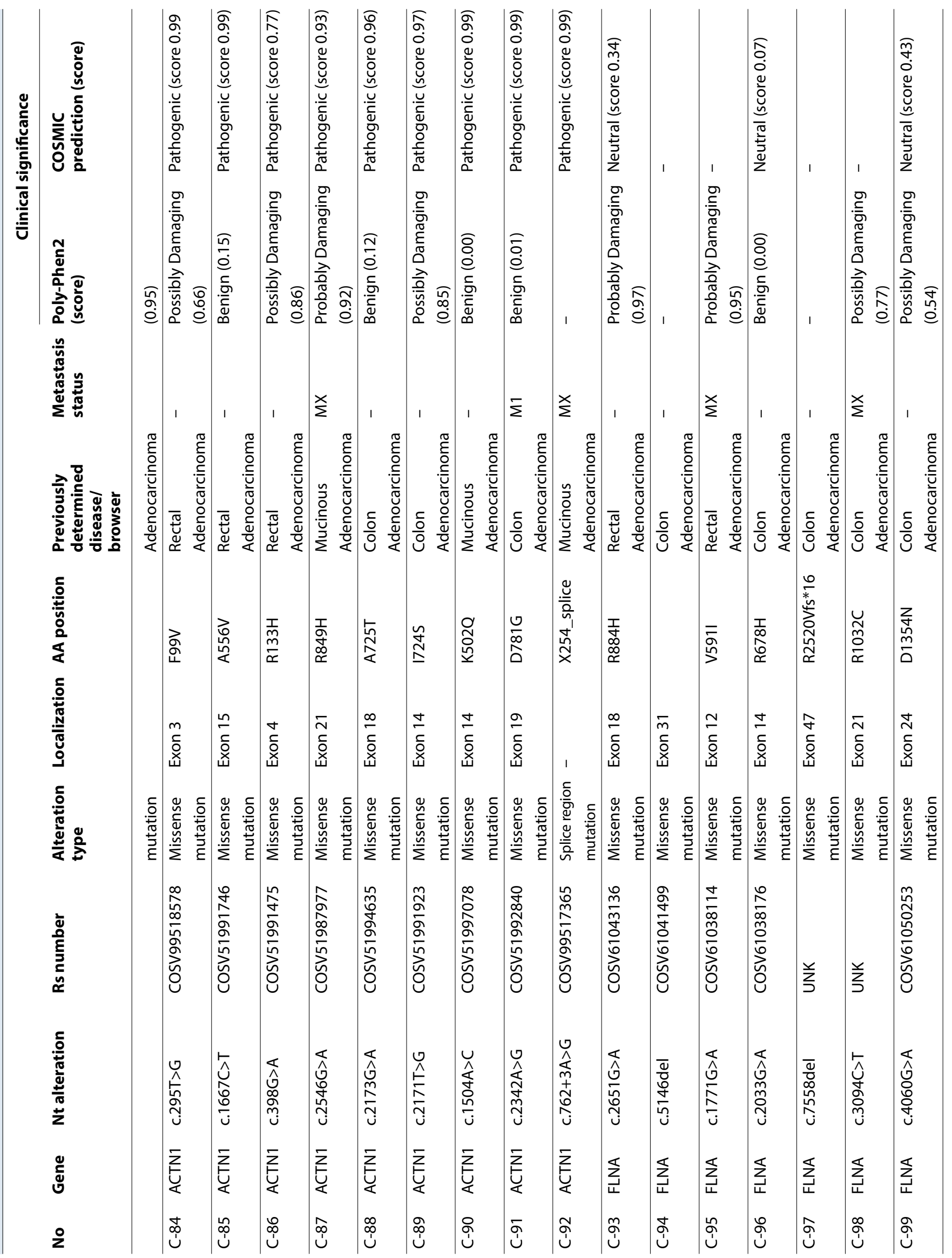




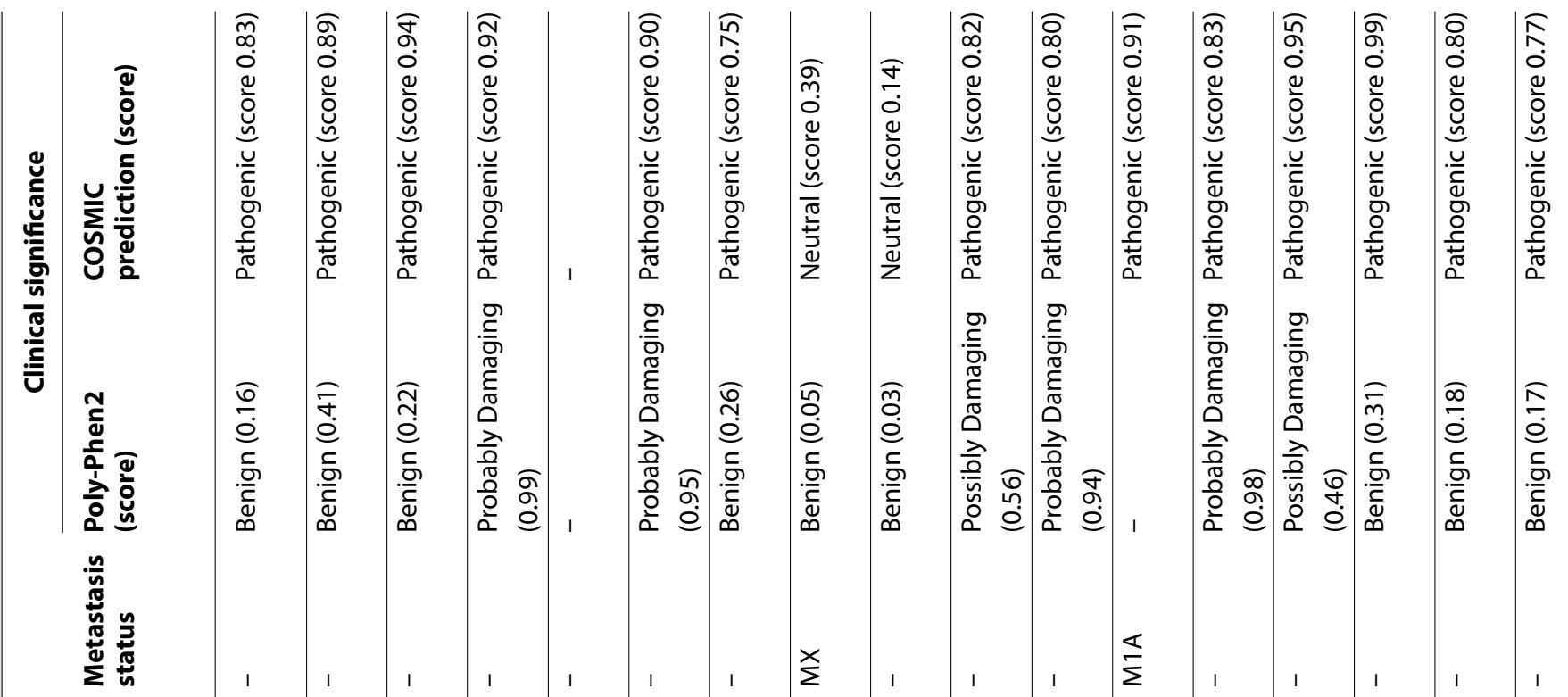

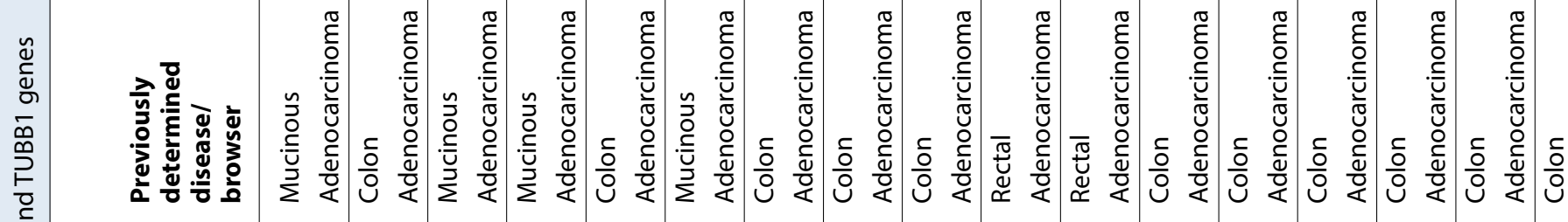

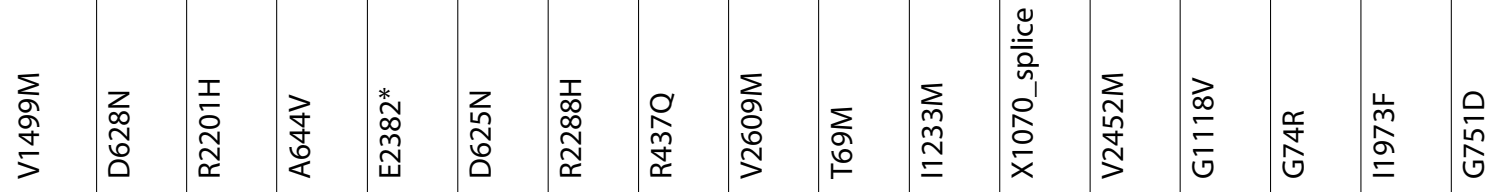

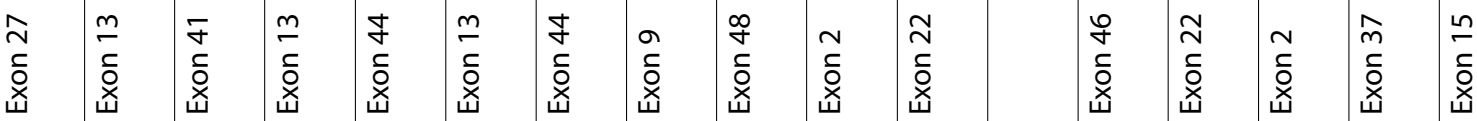

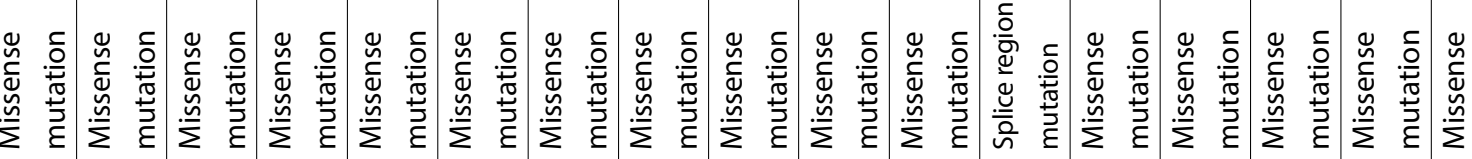

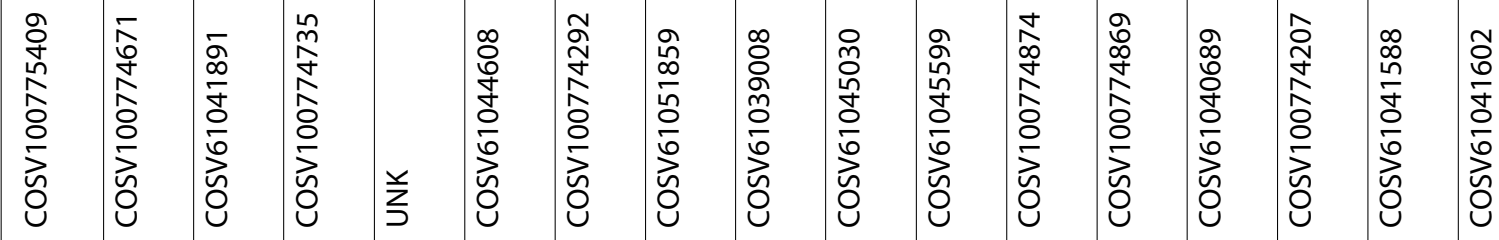

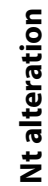

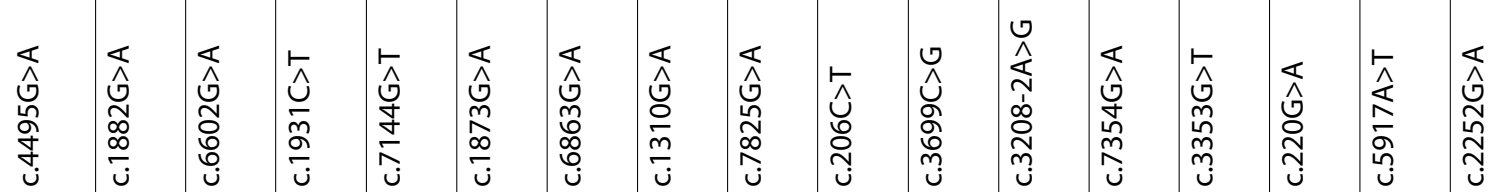

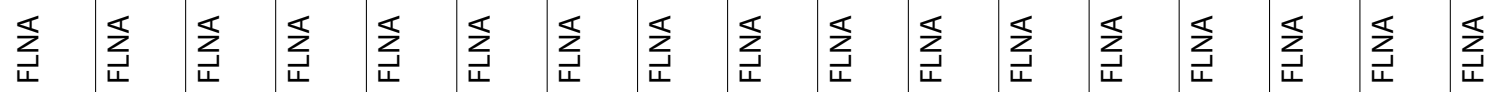

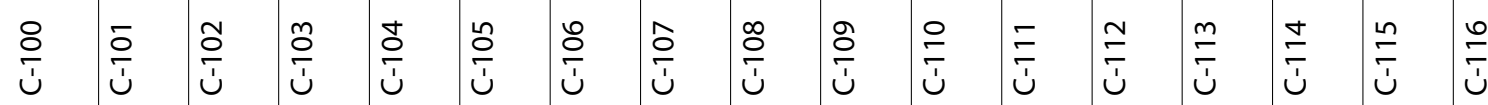




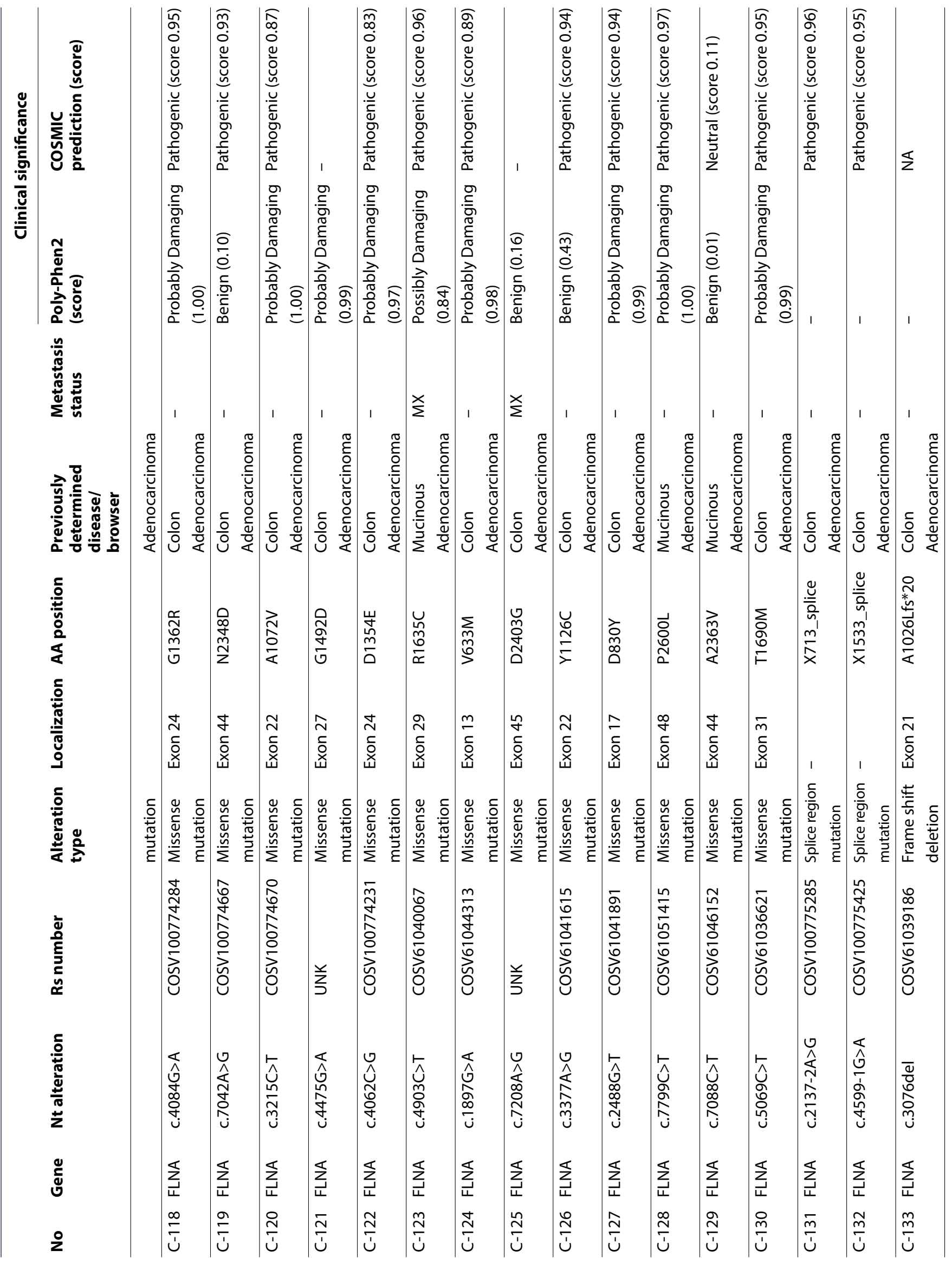




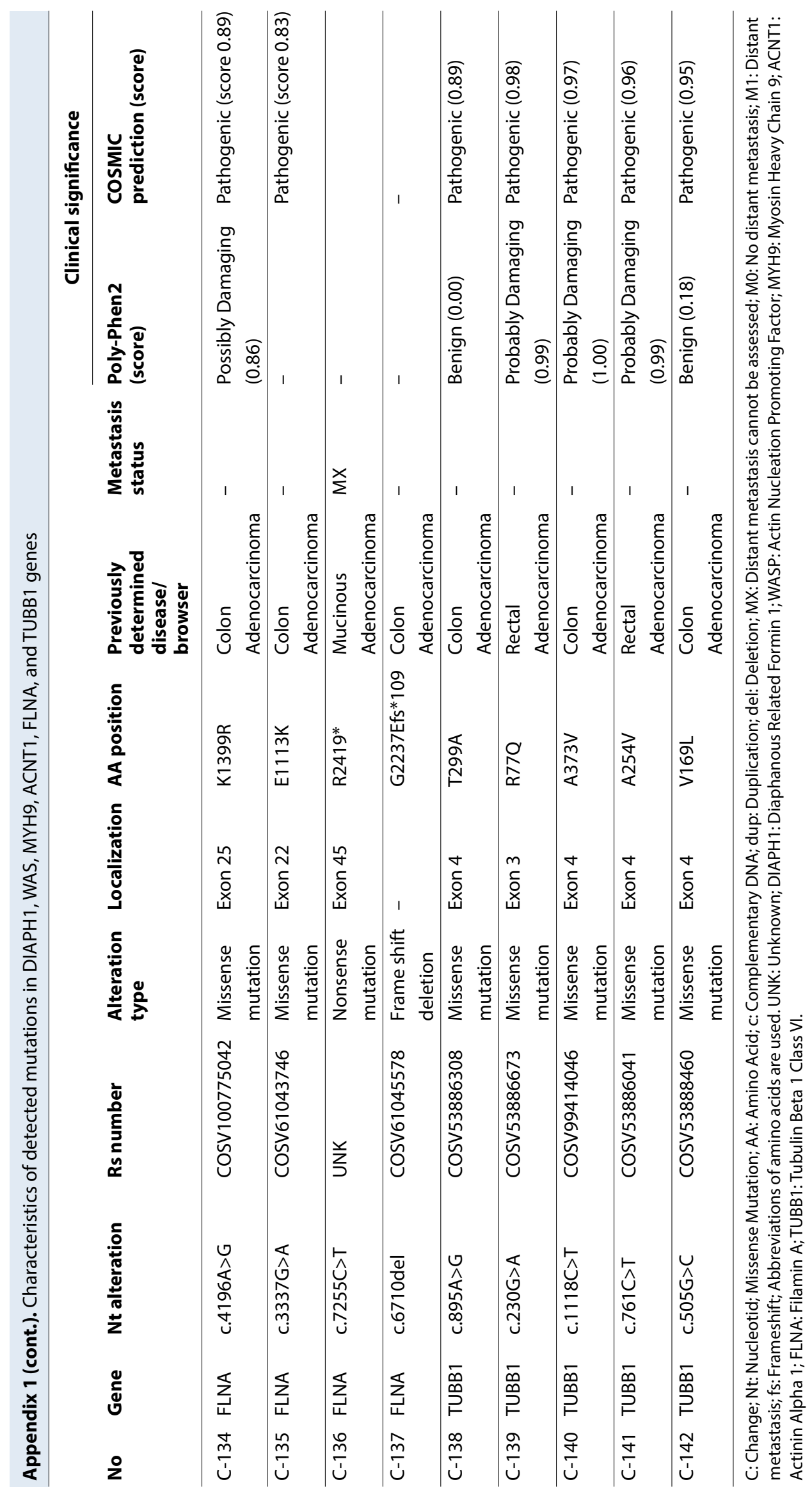

